\title{
EL HUMANISMO EN LA EDUCACIÓN MÉDICA
}

\author{
Jorge Francisco Oseguera Rodríguez \\ Centro de Investigación Educativa y \\ Formación Docente, Querétaro. \\ Instituto Mexicano del Seguro Social, México.
}

Recibido 16-III-2006

Resumen: En los últimos 30 años se ha considerado, de forma creciente, el enfatizar el retorno al humanismo en medicina como una medida para contrarrestar el "corporativismo" que rodea a los sistemas de salud, ya que parece ser el responsable de la deshumanización en la atención médica que hoy se proporciona. Como médicos educadores, nos encontramos preocupados por el impacto que las conductas profesionales de los médicos ejercen sobre el desarrollo de las actitudes y conductas humanistas de los estudiantes y residentes médicos, sin embargo, aún parece existir cierta confusión de lo que implica el término "humanista" en este ámbito. De esto se deriva la necesidad de aclarar lo que entendemos por "humanismo médico" para lograr un acuerdo general que sustente la evaluación del humanismo en los médicos y estar en la posibilidad de plantear estrategias pedagógicas explícitas que permitan impulsarlo y fortalecerlo en el ámbito de la educación y la práctica médica. En este documento se revisa el concepto clásico de humanismo y su evolución dentro de la Medicina hasta considerarse como una forma de vida en la que se estima y se hace énfasis en el bienestar del ser humano y se posibilita la construcción de valores y normas.

Palabras clave: Humanismo, medicina, educación, deshumanización.
Aceptado 25-IV-2006

\section{Introducción}

Los requerimientos de la sociedad, en el siglo XXI, han obligado a las escuelas de educación superior a entrar en procesos de evaluación y acreditación que garanticen que están formando a los profesionales acordes a las necesidades de los ciudadanos de este nuevo siglo. Para lograr esto se ha propuesto como estrategia central el definir las características del egresado que pretenden.

Por otra parte una vez definidas estas características del egresado es necesario evaluarlas desde el punto de vista del desempeño profesional, para garantizar que las escuelas están logrando el profesional deseado. Este desempeño se ha evaluado generalmente desde el punto de vista cognoscitivo y de habilidades, pero poco se han considerado las características humanistas y el compromiso social expresado (Federación Mundial para la Educación Médica, 2000). La evaluación del actuar profesional debe de incluir no sólo los aspectos más rutinarios, de bajo nivel, sino aquellos que se refieren a valores y cualidades personales.

Y aunque la ética y la visión humanista han pasado a formar parte del currículo, los métodos para evaluar a los valores y 


\begin{abstract}
For the last thirty years, medical science has a growing attention regarding an emphasis to return to the humanism to counteract the "corporativism" around the health care system, because it seems the main responsible of the dehumanization of the health are provided. Like medical educators, we are worried about the impact that our own professional behavior exerts on the development of the humanistic attitudes and behaviors of the medical students and residents, however it still seems that the concept "humanism" is confusing among the medical areas. This brings out the need to clarify what we understand by "medical humanism", to reach a general agreement that supports the evaluation of the humanistic medical attitudes and behaviors and outlines specific pedagogical strategies that enhance and strengthen the term in the environment of medical education ad practice. This paper review the classical term "humanism" and its evolution inside the medical science until it may be considered as a way of life with an emphasis made on human welfare and it enables the construction of human values and norms.
\end{abstract}

Key words: Humanism, medicine, education, dehumanization. actitudes de los estudiantes a la hora de abordar problemas de la práctica clínica no han avanzado al mismo.

Es un error pretender evaluar las características humanistas, sin establecer previamente una conceptualización que dé cuerpo a un modelo de la práctica profesional aceptable equivalente al "deber ser" profesional. De esta forma, se establece un "patrón de referencia" contra el cual se compara a cada individuo. El modelo que sirve de "patrón de referencia" en el proceso se construye mediante la articulación del estado del arte necesario para una práctica aceptable de la profesión, con los ambientes de trabajo estándar en los cuales habrá de desempeñarse el individuo, en donde la opinión de los pacientes y las expectativas que tiene la población sobre los profesionales es muy importante, de manera que la educación sea más sensible a las necesidades cambiantes de la sociedad.

Para que el modelo de evaluación resulte funcional deben vencerse dos grandes retos: hacerlo operativo, estableciendo claramente sus componentes, variables e indicadores; traducidos en características claramente enunciadas y determinar los criterios de ejecución, para distinguir el cumplimiento o incumplimiento de las normas.

En el ámbito hispano parlante pocas han sido las instituciones que han definido en forma concreta las características finales de sus alumnos y en muchos casos se han limitado a hacer declaraciones de escasa utilidad para que los estudiantes sepan lo que se espera de ellos al licenciarse, para que los profesores puedan enmarcar sus actividades y mucho menos para que se puedan definir los criterios de evaluación.

La responsabilidad de definir las características de los médicos atañe a diferentes personas de diferentes instituciones. Por ello, definirlas no es una tarea fácil. Es responsabilidad en forma principal de las instituciones educativas, pero otros agentes tienen que ser implicados, entre ellos, los sistemas de salud de cada país, estado, región, etc., los profesionales médicos 
(Baker MZ, Scoffield RH.,1998) y las instituciones que los representan, los recién graduados, los estudiantes, los profesionales de otras profesiones sanitarias (DesMarchais JE., 1999), los representantes de los empleadores, el gobierno, los sindicatos, los pacientes y la sociedad en general (Harden RM., Crosby JR., Davis MH., 1999).

En esta línea, algunas agrupaciones profesionales como el Colegio Americano de Medicina Ocupacional y Ambiental y las facultades de medicina escocesas (Harden RM., Crosby JR., 1999), entre otros, han elaborado inventarios de las características deseadas en los médicos.

En los últimos treinta años, el humanismo en la medicina ha ganado una atención creciente, muchos han llamado a un regreso al humanismo médico como una manera de responder a la transformación corporativa del sistema de cuidado de la salud. Se han alarmado los médicos en lo individual sobre lo que está pasando a sus prácticas que ha llevado a la deshumanización de la atención médica (Nizama-Valladolid, 2002).

Las asociaciones de profesionales están preocupadas por los cambios que la medicina corporativa ocasiona en las responsabilidades de los médicos hacia los pacientes. Los médicos educadores estamos preocupados por el impacto que las conductas de los médicos producen en el desarrollo profesional de estudiantes médicos y residentes (Patiño, 1998, 2000). Pero hoy todavía no todo mundo entiende claramente lo que entendemos por "humanismo" en medicina.

Lo que resalta la necesidad de aclarar lo que entendemos por humanismo médico teniendo como meta el lograr un acuerdo general suficiente para evaluar el humanismo de los médicos y poder plantear intervenciones pedagógicas explícitas que permitan fortalecerlo en el ámbito de la educación y la práctica médica.

Ante este panorama, el interés y el objetivo de este ensayo se centró en revisar el concepto de humanismo y su evolución hasta llegar al concepto actual en el ámbito médico.

\section{El humanismo clásico}

El humanismo ha sido definido de diversas formas. Pero durante el siglo XIII surge la idea de la cultura clásica que puede considerarse como la precursora del humanismo de los siglos XIV y XV, en que se despertó una devoción por la literatura de la antigüedad clásica greco-romana, sin embargo los expertos no han formulado una única definición de los rasgos de esa época, que recoja una aprobación unánime. Esto resulta complicado porque en este periodo se encuentra una modificación del pensamiento filosófico y además también de toda la vida del hombre en sus aspectos sociales, políticos, literarios, científicos y religiosos.

El término humanista a mediados del siglo XV, se utiliza para señalar a quienes enseñaban y cultivaban la gramática, la retórica, la poesía, la historia y la filosofía moral. Humanitas significaba lo que los griegos habían expresado en términos de paideia, es decir, educación y formación del hombre. Considerando a estas disciplinas como las más idóneas para "hacer que el hombre sea aquello que debe ser, de acuerdo a su naturaleza espiritual específica" (Reale y Antiseri, 2001:27).

Alcanza su máximo esplendor en la segunda mitad del siglo XV en donde a los estudios de la litterae humanae se les da un gran valor y se considera que la antigüedad clásica griega y latina son una especie de paradigma y de punto de referencia, en lo que concierne a las actividades espirituales y a la cultura en general y los autores griegos y latinos aparecen como modelos insuperables, como maestros de la humanidad.

Un pionero humanista fue Francesco Petrarca, florentino, muy aficionado a la literatura latina por lo que se dedicó a coleccionar los escritos de estos autores y ésta fue una de las características que permitió la trascendencia de esta corriente filosófica. Él como otros humanistas descubrieron un gran número de manuscritos clásicos, que de otra forma podrían haberse 
perdido, establecieron bibliotecas en las universidades y gracias a la invención de la imprenta difundieron la literatura grecolatina antigua.

Otra razón para la expansión de los intereses humanísticos en Europa se debe a su impacto en las instituciones existentes de educación formal, en donde paulatinamente la mayor parte de las universidades añadieron el programa humanístico de las literaturas griega y latina, como un programa cultural y pedagógico en donde el aristotelismo renacentista utilizó los métodos propios de la escolástica, enriquecidos por el influjo humanista que exigió a los estudiosos el regreso a los textos griegos de Aristóteles y que abandonasen las traducciones latinas medievales y apelasen a los comentarios de otros pensadores griegos. Estos programas fueron ganando aceptación como principio educacional.

La discusión teórica y filosófica acerca del humanismo ha llevado a algunos expertos como Kristeller (en Reale y Antiseri, 2001) a dejar al término el significado técnico que poseía en sus orígenes, limitándolo al ámbito de la retórica y la literatura. De acuerdo a este autor el humanismo de esta época ha sido sobrevalorado, atribuyéndole una función renovadora del pensamiento que no tuvo, pues sólo de manera indirecta se ocupó de la filosofía y de la ciencia. Y es la continuación de la tradición aristotélica medieval, enriquecida por la corriente humanista la que expresaría las verdaderas ideas filosóficas de la época.

Otros autores como Garin, (en Reale y Antiseri, 2001) no opinan lo mismo y consideran que la negación del significado filosófico al humanismo renacentista está en función de entender por filosofía a una construcción sistemática de grandes proporciones y se niega que pueda ser filosofía otro tipo de especulaciones no sistemáticas, problemáticas y pragmáticas. Para él la filosofía humanista presenta una nueva forma de plantearse los problemas con indagaciones concretas, definidas, precisas, en el ámbito de las ciencias morales y en las ciencias naturales, constituyéndose en un filosofar efectivo y auténtico.

De acuerdo a esto lo que el humanismo quería destruir fueron las grandes sistematizaciones lógico-teológicas, una filosofía que subordinaba todos los problemas y todas las investigaciones al problema teológico, filosofía que el humanismo considera como vana, inútil y carente de sentido histórico. Es precisamente este sentido histórico el que permite al humanismo valorar la cultura antigua en su auténtica dimensión: pensamiento de hombres, en una cultura determinada, resultado de sus experiencias y no como oráculos de la naturaleza o de Dios, revelados a los filósofos griegos, sino como productos humanos.

El descubrimiento de lo antiguo permitió a los humanistas el descubrimiento del hombre, adquiriendo el sentido de su propia individualidad y originalidad, el sentido de la creación humana, de la obra terrena y de la responsabilidad, que los llevó a actuar libremente en la vida pública de su tiempo, a la exaltación de la vida civil y de las problemáticas afines a ella, mas allá del oficio literario y del claustro universitario.

Las dos opiniones contrapuestas de estos autores reflejan una discusión que no ha sido resuelta en el ámbito de la filosofía, pero un punto intermedio es considerar que la tradición aristotélica fue transformada al efectuar una nueva lectura de los escritos griegos originales y se le dio un nuevo sentido al hombre y a sus problemas, este nuevo sentido culmina con conceptualizaciones teóricas de la dignidad del hombre como ser extraordinario con respecto a todo el resto del cosmos que se ven manifestadas por las representaciones de la pintura, la escultura y la poesía.

\section{El humanismo en la época moderna}

La concepción de humanismo que el mundo occidental heredó del Renacimiento 
europeo fue la de humanitas, entendida como la acción libre y creativa del ser humano que, al transformar la naturaleza y crear el mundo de la cultura, logra la transformación en el sujeto. Abriendo múltiples posibilidades de desarrollo, convirtiéndolo en un proyecto inacabado, en un individuo único e irrepetible, el cual, mediante su actividad libre e inteligente, se da a si mismo una forma de ser.

Los humanistas volvieron sus ojos a la antigüedad clásica porque vieron en ella el ejemplo de la afirmación de la autonomía del espíritu humano y porque consideraron el ejercicio de la razón como la clave para construir una comunidad plenamente humana.

Hegel (1997) retomó la idea renacentista y describió la unidad dialéctica sujetoobjeto como un proceso de construcción que es a su vez autoconstrucción, en la cual el sujeto al objetivarse, crea el mundo de la cultura y se recupera, mediante su experiencia, como sujeto enriquecido.

Entre la concepción renacentista y otras del siglo XIX como la hegeliana, hay diferencias: el ser humano para los renacentistas es el ser particular, el que al perder su lugar privilegiado en el centro del universo (por efecto de la revolución copernicana) busca constituirse en el centro de los valores. Posición que fue criticada en el siglo XIX por quienes vieron en el individualismo un obstáculo para la justicia social y que interpretaron como un signo de poder que amenazaba la autonomía de las comunidades y como un símbolo de explotación, en perjuicio de la libertad y de las posibilidades de desarrollo de los seres humanos a favor del beneficio de unos cuantos.

El individualismo mostró uno de los límites del humanismo renacentista, pero se expuso a otro límite: la anulación de lo particular en aras de lo universal. Ambos límites se presentan como una tarea ineludible para quien adopta una perspectiva humanista a inicios del siglo XXI, la de resolver la relación contradictoria entre lo universal y lo particular.
Podemos considerar el humanismo, más que una corriente del pensamiento, como una forma de ver la realidad, una perspectiva que considera a la dignidad humana como criterio último de valoraciones y normas y como el horizonte al que deben orientarse las acciones.

El siglo XIX heredó del humanismo y de la ilustración la confianza en el individuo y en la razón. Los avances científicos y tecnológicos hacían suponer un futuro placentero, próspero y saludable, en donde el sujeto era capaz de crear cultura y cambiar el rumbo de la historia. Lo humano era una voluntad incondicionada que hacía del sujeto, el creador de su propio destino. El siglo XX fue la otra cara de la moneda; se avanzó mucho en el conocimiento científico y tecnológico, pero las aplicaciones de éste no siempre han resultado benéficas.

Con la supuesta muerte de las ideologías sobrevino el desencanto, la desesperanza, la incredulidad del ser humano en su razón y en sus posibilidades de transformación. El ser humano no se concibe como libertad autónoma e ilimitada, sino como una persona sujeta a múltiples ataduras y determinaciones. La marca del siglo XX fue el desencanto, el pragmatismo y la barbarie que pone en peligro la vida y que lesiona la dignidad humana.

Es importante entender que lo humano por excelencia no es lo universal que destruye lo particular, ni lo particular que se construye sobre la destrucción de lo universal, sino lo integral que se reconoce en la totalidad.

El humanismo renacentista tiene otra limitante a la que se opusieron los teóricos de la escuela de Frankfurt (Fromm, 1998), la racionalidad funcional, que se fortaleció por efecto de los avances tecnológicos, los descubrimientos geográficos y las conquistas de nuevas tierras, con la consecuente subordinación y explotaron de los habitantes de estas. Y aunque en el humanismo renacentista la dignidad radicaba principalmente en la razón y en la libertad del ser humano, estas cualidades quedaron 
subordinadas al criterio de eficacia y a la obtención del éxito.

El común denominador de la perspectiva humanista es el de considerar a la dignidad humana, por una parte, como un criterio que confiere validez a principios, valores y normas y, por la otra, como horizonte al que han de tender las acciones del ser humano. Se trata de un gran ideal, que incluye otros ideales, pero también de un valor fundamental que sirve de pauta a toda forma de regulación.

Es claro que la especie humana es el resultado de la evolución biológica y por otro lado de su herencia cultural, la que al nacer y desarrollarse en una comunidad le proporciona códigos, usos, costumbres e instituciones y se los ofrece para su apropiación.

Pero la condición humana no se agota en la dimensión biológica ni en la dimensión contextual, ni en la conjunción de ambas, el ser humano es también un ser que acumula experiencia, es un ser que mira al futuro, que tiene la posibilidad de dar significado y sentido a la vida en general, a la propia vida en particular y a la condición humana misma. Que además es capaz de actuar conforme a ese significado y ese sentido para crear cultura y autocrearse como sujeto. Es en esta dimensión de ser proyecto, sujeto en formación, en la que radica la dignidad.

\section{El humanismo médico}

El término humanista es añadido muy frecuentemente al término médico, en una moda tanto imprecisa como intuitiva. La idea del humanismo renacentista en el ámbito médico se resume en lo expresado a fines del siglo XIX por José Letamendi "el que sólo sabe de medicina, ni medicina sabe", "el médico que a la vez no es filósofo, no es ni siquiera médico", ideas que a muchos hace considerar que el médico humanista es aquel que cultiva las artes, la pintura, la literatura, "que cultiva las ciencias que enriquecen el espíritu" (Maria Moliner, 1983), pero el humanismo es más que sólo cultivar estas artes, como hemos visto, y si bien es importante para el médico conocer esas disciplinas, no es esencial para su profesión.

La idea del humanismo médico se encuentra ya expresada en el juramento y en otros libros del Corpus Hipocrático, durante estos últimos años, gran parte de los profesionales de la medicina, han perdido la imagen humanista que los identificó durante milenios, por lo que hoy son considerados como simples técnicos, ávidos de reconocimiento económico y profesional, adheridos al modelo racional cientificista y divorciados de la sensibilidad humana (Mainetti, 1992).

Pero, la ciencia no es la causante de tal reduccionismo materialista sino algunos científicos que sólo quieren saber de ciencia. Hoy creo que todos estamos de acuerdo en que el hombre no es una máquina ni la enfermedad una simple descompostura; también, en que la medicina no es una ciencia sino que, como lo aclara Edmund Pellegrino (1990): “...es la más científica de las artes, la más artística de las humanidades, la más humanista de las ciencias." Lo que le confiere un status epistemológico propio y diferente a las demás disciplinas.

El acto médico es un ejercicio de humanismo, entendido también como humanitarismo. Que se basa en una insustituible relación de confianza mutua entre el médico y su paciente, cuyas dimensiones humanas y de espiritualidad deben ser individualmente consideradas y atendidas.

El humanismo, dentro del ámbito médico, nutre y se nutre de principios y normas que constituyen el dominio de la ética médica. En los últimos tiempos, la bioética ha adquirido gran interés, quizá como respuesta a la preocupación, cada vez mayor, que ocasionan los cambios debidos al auge de la medicina institucional y a la creciente aplicación de innovaciones tecnológicas en el campo de la salud, situaciones ambas que tienden a despersonalizar la 
práctica médica y que constituyen lo que se ha dado a denominar la deshumanización de la medicina.

Esta realidad hace necesaria plantear nuevas perspectivas dentro del humanismo médico. Ahora bien, no se trata sólo de escudriñar en la relación entre un médico y su paciente; es necesario hurgar en la interacción del médico con la colectividad, de la institución de salud con el enfermo y de los médicos con los gobiernos y los usuarios de los servicios. Y sobre todo, el reconsiderar el papel que están jugando las instituciones formadoras de médicos en el cumplimiento de las demandas del ser individual y colectivo.

Partiendo del análisis de los perfiles de egreso declarados por diversas escuelas de medicina en donde se plantea la necesidad de la formación humanista del médico (De la Fuente, 1994), es necesario primero aclarar cual es el concepto de "médico humanista", entendiendo que lo humano, mas allá del basamento biológico, es lo que nos hace diferentes de los animales. Es toda la carga de racionalidad, de civilización y cultura, de historia o religión que cada uno quiera depositar en el concepto.

Así pues, entendemos por humanismo médico, a todo el conjunto de valores, actitudes y prácticas que promueven una auténtica vocación de servicio y dan lugar a considerar al paciente como un semejante que sufre y solicita alivio. Los aspectos más significativos que promueven el humanismo en el trato con los pacientes son: el afecto, el apoyo, el respeto y la solidaridad, que, a la vez, son los que nos procuran la mayor cooperación posible del paciente para conocerlo mejor y ayudarlo más (Viniegra, 2000).

Se han establecido algunas características del médico humanista como son: la empatía, la autenticidad, la compasión, la fidelidad, la integridad, el respeto, la espiritualidad y la virtud; tratando de diferenciarlos del profesionalismo en donde otros autores como Markakis (2000) han agrupado características como son: la responsabilidad, el altruismo, el compromiso con la excelencia, el deber, el honor y el respeto para otros.

Consideramos que la dimensión humana es más amplia e integradora y no es posible fragmentarla, compartimos las categorías establecidas por Rogers (2000):

1. El respeto de los puntos de vista del paciente y la consideración de cada una de sus opiniones en la toma de decisiones respecto a su salud.

2. El atender el bienestar psicológico del paciente.

3. Recordar que el paciente es único e individual.

4. Tratar al paciente en su contexto familiar y ambiente social y físico.

5. Poseer buenas cualidades de comunicación y para escuchar.

6. Proporcionar confidencialidad y confianza.

7. Demostrar calidez y compasión, y

8. Ser empático.

Estas categorías son compatibles con los rasgos de una atención humanizada expresados por Gafo (1993): Reconocimiento de la dignidad intrínseca de todo enfermo, unicidad del paciente, globalidad del paciente, el respeto a la libertad, la participación de los pacientes, el igualitarismo y el equilibrio en la relación empatía-afecto.

Y también son coincidentes con lo planteado como derechos del enfermo y los pacientes (el derecho a la vida, a la salud, a la autonomía, a la información, a la verdad, confidencialidad, a la intimidad, a la libertad y al trato digno) que se han incorporado a diversos marcos legales en el mundo e Hispanoamérica: Declaración universal de los derechos humanos, 1948; Derechos de los Pacientes, Asociación Medica Mundial, 1995; Resolución 13437 del Ministerio de Salud Colombiano, 1991; Ley 8239 sobre los derecho y deberes de los usuarios de los servicios de salud en Costa Rica, 2002; etc. (Tena Tamayo, 2002). 
La ausencia de esas características en el personal de salud, además de diversos factores, ha favorecido la deshumanización en la atención médica. Término utilizado cuando se trata al paciente como un objeto y no se consideran sus rasgos personales e individuales, prescindiendo de sus sentimientos y valores, "cosificándolo" como el paciente de la cama X, o el paciente con tal o cual padecimiento, relegando a un segundo plano sus dimensiones personales, siempre específicas en cada paciente. También cuando existe la indiferencia o frialdad en la interacción humana, una preocupación distante que constituye una negación de humanidad hacia el enfermo en el propio médico (Gafo, 1993).

Estas situaciones se presentan en un sistema dominado por instituciones en donde las exigencias de productividad, eficiencia y rentabilidad son prioritarias. Las instituciones prestadoras de servicios de salud procuran el control en busca de la máxima utilidad o el máximo ahorro por encima de todo lo demás, generando ambientes más o menos degradantes de la condición humana, en donde las instituciones limitan la reflexión sobre la experiencia y los acontecimientos, limitando al profesional de la salud a una ejecución técnica y no reflexiva de su actividad profesional. Es aquí en donde algunos profesionales muestran una auténtica falta de vocación de servicio y la incapacidad para sobreponerse a estas condiciones imperantes, perpetuando estos ambientes degradados y deshumanizados (Viniegra, 2005).

Otro signo de deshumanización es la restricción de la libertad, la falta de autonomía del enfermo en donde éste no es protagonista de su destino, en donde no se le hace partícipe de la toma de decisiones que ponen en juego lo más valioso que tiene su vida, algunas veces argumentando su falta de capacidad, con una visión paternalista, no siempre bien justificada. Procurar la autonomía incluye aspectos como el decidir quien será su médico tratante, cuándo desea hospitalizarse u operarse, el derecho a ser informado de su diagnóstico, del por qué de su tratamiento y del pronóstico de su enfermedad. Y hasta la decisión de en qué momento morir.

La deshumanización de la medicina tanto en sus características globales como individuales, tiene que ser abordada desde una perspectiva social e histórica que analice los diferentes factores involucrados sin la cual no es posible vislumbrar encontrar soluciones adecuadas.

Cabe hacer notar que este proceso deshumanizador se presenta en una sociedad con una desbordada criminalidad, en incontrolable delincuencia, con flagrantes injusticias; nuevas guerras y muertes por viejos y nuevos conflictos de interés, religiosos o nacionalistas; que desarrollan nuevos procesos de exclusión y de odio. Dentro de una sociedad materializada, crecientemente desprovista de valores fundamentales para una convivencia humana satisfactoria, presa de la desconfianza y de la desesperanza, sentimientos que son muchas veces fabricados desde los centros de decisión mediática, o simplemente aprovechados para hacer de la confusión, real o provocada, el mejor de los negocios.

Con el desarrollo científico y tecnológico cuyas manifestaciones más importantes se dieron en la primera mitad del siglo XX se ha producido la socialización de la medicina, en donde las grandes instituciones de salud como representantes del estado benefactor han llevado la atención a colectividades, sindicatos, comunidades, etc. Es un modelo de prestación de servicios que partiendo de principios de "justicia social" ha llegado en nuestros días a la visión gerencial de la industria de la atención de la salud, rompiendo con la relación individual entre el médico y su paciente, originando conflictos en esta relación individual, socializada en donde se gesta el proceso deshumanizador.

La deshumanización no es un problema particular de la medicina, sino parte de un todo que es la deshumanización de la sociedad, producto de la "modernización 
reflexiva", entendiendo por esto la tesis planteada por Beck, en la cual los sujetos (personas o instituciones) a través de conocimiento, pero paradójicamente, el no conocimiento por falta de reflexión en el mismo, evolucionan produciendo individualización y causando crisis en las estructuras sociales, las instituciones, la familia y los seres en lo individual (Beck, Giddens y Lash, 1997).

Así pues, los mismos condicionantes sociales que originan deshumanización en la prensa, en el arte, en los medios económicos y políticos contribuyen a la presencia de este fenómeno en la asistencia médica. Es aquí donde la capacidad individual de un médico no es suficiente para compensar las deficiencias o limitaciones que la institución le impone.

Son estos sistemas de atención médica, que han transformado a los profesionales de la salud en un "manufacturero de la salud", los ambientes académicos en donde los futuros profesionales se moldean y aprenden los esquemas que han de repetir, perpetuando los modelos que muchos criticamos pero que no hemos encontrado la forma de cambiarlos.

En el ámbito de las instituciones los responsables de esta imagen deshumanizada no son únicamente los médicos, son también el resto de los sujetos que participan en el proceso de atención. Desde los administradores que dictan las políticas a seguir (incluso los supranacionales como el Fondo Monetario Internacional), los encargados de la recepción de los pacientes que pueden no ofrecer un trato adecuado, hasta cada uno de los sujetos que directa o indirectamente participa en la atención médica como puede ser un camillero o intendente.

Es aquí donde la reflexión individual, la reflexión de las instituciones hace crisis, donde el ser individual ya no conoce qué es lo que quiere, es en este marco del inicio del siglo XXI que las instituciones de salud a nivel mundial están sufriendo una crisis sin precedentes y sólo una mayor reflexión, más expertos, más ciencia, más esfera pública, más autoconciencia y más autocrítica abrirán nuevas y mejores posibilidades para este mundo (Beck, Giddens y Lash, 1997).

Otro de los factores que favorece la deshumanización ha sido el desarrollo tecnológico que ha hecho, aparentemente, más fácil para lograr un diagnóstico el solicitar diversos estudios (tomografías, ultrasonidos, baterías de exámenes, etc.), que establecer una relación médico paciente personalizada, con un diálogo y una exploración adecuada, ese desarrollo que nos ha llevado a olvidarnos de los más elementales principios de la medicina clínica. Estos principios en este momento están siendo revalorados y se presentan como una alternativa para enfrentar el excesivo uso de la tecnología que fragmenta al ser humano y es causa de la dependencia social y económica, que está mediada por intereses muy diversos y casi siempre ajenos a los verdaderos intereses que tratan de lograr una mejor atención médica.

Los factores económicos son los que mueven el mundo, o los factores políticos originados en esos intereses económicos, pero finalmente estos intereses económicopolíticos deben de ser considerados como intereses de grupo, en una interrelación circular en la cual los cambios en la ética individual genera cambios en la ética social y esta, a su vez en los seres en lo particular generando un círculo, difícil de romper sin una intervención intencionada, que requiere una profunda reflexión.

\section{La educación humanista en medicina}

Tradicionalmente la enseñanza de la medicina consistía en la relación directa del aprendiz y el maestro, el cual proporcionaba los diferentes secretos de la disciplina de manera gradual y el alumno se desarrollaba estrechamente vinculado a la práctica, trasmitiéndose el conocimiento de generación en generación. 
Actualmente la medicina se enseña en las universidades y en las unidades de atención médica en un contexto social que se aleja cada vez más del modelo tutorial tradicional, condicionado esto por los mismos cambios que ha tenido la atención médica, la cual tradicionalmente también correspondía a la relación entre dos personas (el médico y el paciente) y actualmente consiste en la relación entre las instituciones de salud y los diversos grupos sociales.

El modelo dominante de educación médica tuvo su nacimiento en el famoso informe Flexner, que orientó la medicina académica. Este modelo ofrece las siguientes características: a) Reduccionismo biológico (desde el punto de vista del objeto de la medicina. Es el llamado modelo biomédico, dirigido a la patología general y a la enfermedad, sólo referido al cuerpo humano como objeto propio del conocimiento médico). b) Conocimiento científico-natural (desde el punto de vista del método de la medicina. Es el paradigma clínico tradicional, descriptivo-explicativo de las especies morbosas o entidades nosológicas a las que se refiere el diagnóstico del enfermo como caso individual). c) Naturalismo normativo (desde el punto de vista de la axiología y la ética, el esquema terapéutico clásico se justifica por el principio de beneficencia y se fundamenta en el orden natural o "fisiológico", cuya preservación y restauración constituye el sentido y deber ser del acto médico).

En contraposición el modelo humanista aboga por los siguientes principios: $a$ ) Holismo (realidad biopsicosocial, concepción pluridimensional y multicausal de salud y enfermedad en términos de bienestarmalestar). b) Hermenéutica o conocimiento interpretativo (la relación interpersonal médico-paciente como contexto, la historia clínica centrada en el enfermo antes bien que en la enfermedad) y c) Normativismo o moral de la libertad (la autonomía de las normas respecto de la naturaleza de la cosas, y la conducta terapéutica como posibilidad de mejora de la condición humana (Mainetti, 1992).
Estamos claros en que estudiamos al ser humano, que debe ser abordado en su totalidad, para promover su desarrollo integral. La perspectiva holística pretende entender un fenómeno en su carácter de totalidad, como un sistema abierto que configura sus diferentes componentes, estructuras y procesos en organizaciones dinámicas (percepción, cognición, valores, metas, motivos, factores biológicos, etc.), asumiendo la posición de buscar las regularidades y los esquemas unificadores contenidos en la libre expresión de los sujetos.

Esta visión emergente demanda una ciencia diferente, basada en una nueva comprensión del universo y de quiénes somos; no puede separar la ciencia de la espiritualidad, sino que debe integrarlas en un marco ampliado de la experiencia humana. Pretende lograr un proceso de reestructuración e innovación en las estructuras sociales, políticas y económicas, como una alternativa a un modelo de civilización que se ha convertido en una fuente de problemas (Gallegos, 1999).

La visión holística reclama la evaluación del estudiante en su integridad y en su contexto. Tendencia que surge como alternativa a la fragmentación del aprendizaje (y de su sujeto) en ámbitos o esferas cognitivas, afectivas y psicomotoras, despojadas de todo sentido personal. Además, trata de aproximar a la realidad el acto evaluativo y analizar de manera natural el proceso didáctico, aportando y valorando información a partir de las prácticas cotidianas de trabajo, de la realización de las tareas docentes, de la comunicación entre los participantes, a fin de orientar, regular y promover el aprendizaje.

Por lo tanto, lo primero que tenemos que considerar es si el currículo, es el adecuado para formar a los médicos en los principios éticos y humanistas deseados y por otra parte considerar el currículo oculto que se hace manifiesto en la práctica cotidiana, que es donde se aprende realmente la clínica y en la cual los sistemas institucionales más allá de las posturas individuales de los 
profesores, se hacen manifiestas y moldean al futuro profesional.

En necesario considerar que en este proceso deshumanizador que ha sufrido la sociedad en general y en particular la medicina, participa, de manera importante, el modelo dominante de educación que privilegia el aprendizaje memorístico, la acumulación del conocimiento en la cual al sujeto se le enseña ha obtener información y acumularla, sin necesariamente, aprender el proceso de la reflexión que le permita hacer concientes sus creencias y convicciones, las cuales generalmente se encuentran ocultas, para que pueda reconocer que detrás de su forma de actuar está su forma de ver el mundo y sus propios intereses, y al reconocer lo que hace y porqué lo hace estar en condiciones de contrastarlo con otros puntos de vista, cuestionarse y tener una introspección autocrítica que le permita generar un conocimiento reflexivo con el cual genere una postura personal ante la realidad (Viniegra, 1999).

Es necesario que el modelo educativo dominante se modifique fomentando la práctica de la reflexión sobre la experiencia, que permita producir un conocimiento crítico, transformador del ser individual, que a su vez sea generador de un cambio en su entorno. Se requiere, entonces, la acción intencionada de las instituciones educativas para romper este círculo deshumanizador, acrítico. Estas intervenciones presuponen la necesidad de un cambio en el modelo educativo partiendo no sólo de modificaciones al currículo, sino ejerciendo acciones dirigidas a generar la toma de una postura reflexiva por parte de los docentes, sin olvidar la importancia de los ambientes en donde se lleva a cabo el proceso educativo.

Este último punto, el de los ambientes, es trascendente en la educación médica puesto que las áreas de asistencia, hospitales y clínicas de atención, son los ambientes en donde se lleva a cabo el proceso de formación de los futuros médicos y especialistas. En donde es necesario romper el círculo, necesitamos cambiar el modelo de atención médica, para transformar el modelo educativo de manera paralela. No es posible tener una buena atención médica si no tenemos una buena educación médica y viceversa.

\section{Conclusión}

El humanismo en medicina es una forma de vida en la que se valora y se hace énfasis en el bienestar del ser humano. Favoreciendo el que asumamos la conciencia de nosotros mismos, como individuos y como especie. $\mathrm{Y}$ permite la convicción de que todo individuo posee una especial dignidad, por lo que no puede ser utilizado como medio, ni reducido a instrumento, ni esclavizado, ni explotado por otros hombres. Y posibilita la construcción de valores y normas que protegen esa dignidad (Soberon, 1994).

La medicina, la educación y la sociedad misma se encuentran hoy en una crisis de identidad respecto de su objeto, método y fin, que se expresa en un conflicto de paradigmas o modelos de racionalidad. Y aunque el modelo positivista continúa siendo el modelo dominante, empiezan a valorarse las posibilidades del modelo humanista. Los cambios necesarios para transformar la educación y la atención médica para modificar el llamado proceso deshumanizador dependen de las decisiones sociales y políticas que deberían tomarse tras un amplio proceso de deliberación, en que intervinieran la totalidad de los afectados por ellas. Sería la manera de legitimar moralmente esas decisiones, de hacerlas realmente justas, válidas, legítimas.

Es aquí entonces, en donde la acción pedagógica dirigida a crear una actitud crítica que permita reflexionar en la importancia de transformar los ambientes educativos nos permitirá iniciar un cambio en la atención medica dirigida a la rehumanización del actuar cotidiano del médico, aunque esto no será posible sin una "modernización reflexiva" de la sociedad en 
su conjunto, pues como hemos enfatizado, la llamada deshumanización de la medicina, es la pérdida de los valores del grupo de los profesionales de la salud (enfermeras, estomatólogos, recepcionistas, técnicos, trabajadores sociales, etc.) y de la sociedad en su conjunto (industria farmacéutica, aseguradoras, asociaciones gremiales), en la cual están inmiscuidos muchos conflictos de intereses.

El resolver los conflictos de intereses solo será posible a través de la reflexión individual y colectiva que dirija estos intereses hacia el bienestar humano y más allá de éste hacia el bienestar ecológico, es necesario considerar una visión integral holística, que permita transformar nuestro pensar en el actuar y nuestro discurso en la acción y la reflexión cotidiana de nuestro vivir.

\section{Referencias bibliográficas}

Baker, Mary; Scoffield, Hal. (1998.) "Educational needs of internal medicina residency graduates: general internist versus subspecialists". Medical Education. Vol. 32, No 5. USA.

Beck Ulrich, Giddens; Anthony, Lash Scott. (1997). Modernización reflexiva. Política, tradición y estética en el orden social moderno. Madrid. Alianza Editorial.

De la Fuente, Ramón; Piña García, E.; Gutiérrez Ávila, H. (1994). "La formación del médico del siglo XXI y el plan único de estudios de la Facultad de medicina de la Universidad Autónoma de México". Educación Médica y Salud Vol. 28, No 3. México.

Des-Marchais, Jacques. (1999). "A Delphi Technique to identify and evaluate criteria for construction of PBL problems". Medical Education Vol. 33, No. 7.
Federación Mundial para la Educación Médica. (2000). "Cumbre Mundial de Educación Médica. Declaración de Edimburgo 1993". Revista Cubana Educación Médica Superior Vol. 14, No 3. Cuba.

Fromm, Erich. (1998). El humanismo como utopía real. Barcelona. Ediciones Paidós Ibérica.

Gallegos, Ramón. (1999). Educación holística, México, Pax-México.

Gafo Fernández, Javier. (1993). 10 palabras clave en Bioética. Navarra, España. Verbo Divino.

Hegel Georg Wilhelm Friedrich. (1997). Enciclopedia de las ciencias filosóficas. Madrid. Alianza Editorial.

Harden, R.M.; Crosby, J.R.; Davis, M.H. (1999). "An introduction to outcomebased education". AMEE Education guide. Outcome-based education. Vol. 14.

Harden, RM.; Crosby, J.R.; Davis, M.H.; Friedman, M. "From competency to meta-competency: a model for the specification of learning outcomes". AMEE Education Guide. Vol. 14. 1999.

Markakis Kathryn, Beckman Howard; Suchman Anthony; Frankel, Richard. (2000). "The Path of Professionalism Cultivating Humanistic Values and Attitudes in Residency Training". Academic Medicine. Vol. 75. USA.

Mainetti, José. (1992). La transformación de la medicina. La Plata, Argentina.

Maria, Moliner: (1983). Diccionario del uso del español. Madrid. España: Editorial Gredos. 
Nizama-Valladolid, Martín. (2002). "Humanismo Médico". Revista de la Sociedad Peruana de Medicina Interna. Vol. 15. No.1. Perú.

Patiño, José. (1998). "Discurso de posesión del Académico José Félix Patiño Restrepo como Presidente de la Academia Nacional de Medicina". Revista Colombiana de Cirugía Vol. 13. Colombia.

Patiño, José. (1993). "Educación médica en el año 2000”. Tribuna Médica Vol. 88. Colombia.

Pellegrino, Edmund. (1990). "The medical profession as a moral community". Bulletin of the New York Academy of Medicine Vol. 66, No. 3. USA.

Reale Giovanni, Antiseri Dario. (2001). Historia del pensamiento filosófico y científico. Barcelona. Editorial Herder.

Rogers John, Coutts Louisa. (2000). "Do Students' Attitudes during Preclinical Years Predict Their Humanism as Clerkship Students?" Academic
Medicine Vol. 75, No. 10. S74-S77. USA.

Soberon, Guillermo; García-Viveros, Mariano; Narro-Robles, José. (1994). "Nuevos frentes del humanismo en la practica medica". Salud Pública de México, Vol. 36. México.

Tena Tamayo, Carlos; Ruelas Barajas, Enrique; Sánchez González, Jorge. (2002). "Derechos de los pacientes". Revista Medica del IMSS Vol. 40, No. 6. México.

Viniegra, Leonardo; Aguilar, Estela. (1999). Hacia otra concepción del currículo, un camino alternativo a la formación de investigadores. México. Instituto Mexicano del Seguro Social.

Viniegra, Leonardo. (2000). La investigación en la educación. Papel de la teoría y la observación. México. Instituto Mexicano del Seguro Social.

Viniegra, Leonardo. (2005). "El desafío de la educación en el IMSS: cómo constituirse en la avanzada de la superación institucional". Revista Médica del IMSS Vol. 43 No. 4. México. 
